\title{
All-Optical Production of a Degenerate Fermi Gas
}

\author{
S. R. Granade, M. E. Gehm, K. M. O'Hara, and J. E. Thomas \\ Physics Department, Duke University, Durham, North Carolina 27708-0305
}

(October 30, 2018)

\begin{abstract}
We achieve degeneracy in a mixture of the two lowest hyperfine states of ${ }^{6} \mathrm{Li}$ by direct evaporation in a $\mathrm{CO}_{2}$ laser trap, yielding the first all-optically produced degenerate Fermi gas. More than $10^{5}$ atoms are confined at temperatures below $4 \mu \mathrm{K}$ at full trap depth, where the Fermi temperature for each state is $8 \mu \mathrm{K}$. This degenerate two-component mixture is ideal for exploring mechanisms of superconductivity ranging from Cooper pairing to Bose condensation of strongly bound pairs.
\end{abstract}

PACS numbers: $32.80 . \mathrm{Pj}$

Degenerate two-component Fermi gases offer tantalizing possibilities for precision studies of pairing interactions in systems for which the density, temperature, and interaction strength are widely variable. Of particular interest are certain two-component mixtures of ${ }^{40} \mathrm{~K}$ and ${ }^{6} \mathrm{Li}$ which exhibit magnetically tunable Feshbach resonances, enabling variation of the s-wave scattering interaction from strongly repulsive to strongly attractive. Attractive mixtures in these systems are analogs of superconductors, since they have been predicted to undergo a superfluid transition as a result of Cooper pairing at experimentally accessible temperatures [1.2]. Recently, two groups have predicted the possibility of superfluidity arising from strong pairing in the vicinity of the Feshbach resonance 3,4]. Transition temperatures of up to half the Fermi temperature are predicted to result from the strong coupling of the two-state Fermi gas to the bosonic molecular state which causes the resonance. Since most high temperature superconductors achieve transition temperatures of only a few percent of the Fermi temperature, two-state Fermi gases may be the highest temperature Fermi superfluids ever studied [5]. Further, these systems may permit observation of the transition from weak Bardeen-Cooper-Schrieffer superfluidity to Bose condensation of strongly-bound pairs [6].

In contrast to Bose-Einstein condensates, which can be prepared and studied in magnetic traps, two-component Fermi superfluids must be prepared in state-independent optical dipole traps, since the required pairs of hyperfine states in ${ }^{6} \mathrm{Li}$ and ${ }^{40} \mathrm{~K}$ are high-field seeking [1,2,7]. A degenerate Fermi gas has been produced by direct evaporation of a two-state mixture of ${ }^{40} \mathrm{~K}$ in a magnetic trap, using a dual radio-frequency-knife method [8]. Sympathetic cooling of fermionic ${ }^{6} \mathrm{Li}$ to degeneracy also has been achieved by using mixtures of ${ }^{6} \mathrm{Li}$ with bosonic ${ }^{7} \mathrm{Li}$ in a magnetic trap [9,10]. However, to explore superfluidity in these systems, transfer to an optical trap and subsequent state preparation is required. The procedure for preparing an optically trapped two-state Fermi gas can be greatly simplified by direct evaporation in an optical trap.

In this Letter, we demonstrate all-optical production of a degenerate mixture of the two lowest hyperfine states of fermionic ${ }^{6} \mathrm{Li}$ in a stable, $\mathrm{CO}_{2}$ laser trap [11]. The trap is loaded from a magneto-optical trap (MOT) at an initial temperature of $150 \mu \mathrm{K}$. Degeneracy is obtained by forced evaporation, accomplished by continuously lowering the trap depth; the trap is then adiabatically recompressed to full depth. At this stage, more than $10^{5}$ atoms remain at temperatures below $4 \mu \mathrm{K}$, less than half of the Fermi temperature of $8 \mu \mathrm{K}$. These results are consistent with scaling laws we have derived for the phase-space density as a function of trap depth [12].

Our ${ }^{6} \mathrm{Li}$ experiments employ a $\mathrm{CO}_{2}$ laser trap with a single focused beam, rather than a crossed-beam geometry as used recently to produce a ${ }^{87} \mathrm{Rb}$ Bose-Einstein condenstate (BEC) by forced evaporation 13. Nevertheless, after free evaporation at full trap depth, we achieve a very high initial phase-space density of $\simeq 8 \times 10^{-3}$, somewhat larger than that obtained after free evaporation in the ${ }^{87} \mathrm{Rb}$ BEC experiments.

A commercial, radio-frequency-excited $\mathrm{CO}_{2}$ laser (Coherent-DEOS LC100-NV) provides $140 \mathrm{~W}$ at $\lambda=$ $10.6 \mu \mathrm{m}$ for the trapping laser beam. An Agilent (6573A) power supply produces stable current for the radiofrequency source, yielding a very stable laser intensity. The laser output is deflected by an acousto-optic (A/O) modulator to control the power. A cylindrical ZnSe telescope corrects the output of the A/O for ellipticity, and the beam is expanded by a factor of 10 before passing through an aspherical $19.5 \mathrm{~cm}$ focal length lens. This lens focuses the beam into the vacuum system, yielding a $1 / e^{2}$ intensity radius of $47 \mu \mathrm{m}$. The corresponding Rayleigh length is $z_{0}=660 \mu \mathrm{m}$. With an incident power of $65 \mathrm{~W}$ in the trap region, the trap depth is estimated to be $690 \mu \mathrm{K}$. The corresponding radial (axial) oscillation frequency for ${ }^{6} \mathrm{Li}$ is predicted to be $6.6 \mathrm{kHz}(340 \mathrm{~Hz})$, with a geometric mean of $\nu=\left(\nu_{x} \nu_{y} \nu_{z}\right)^{1 / 3}=2400 \mathrm{~Hz}$.

The radial oscillation frequency is measured by modulating the frequency of the A/O to produce a sinusoidal displacement at the trap focus with an amplitude of 0.2 $\mu \mathrm{m}$. After the sample is initially prepared at a temperature of $\simeq 15 \mu \mathrm{K}$, the modulation is applied for one second. The number of remaining atoms is measured by resonance fluorescence. Repeating this procedure as a function of modulation frequency reveals a resonance in 
the trap loss at $6.5 \mathrm{kHz}$, in close agreement with predictions. Parametric resonance methods 14 yield results consistent with the expected radial and axial oscillation frequencies after correction for the expected resonance frequency shift 15].

Extremely low residual heating rates are attained in the experiments. At the maximum trap intensity of 1.9 $\mathrm{MW} / \mathrm{cm}^{2}$, the optical scattering rate is 2 photons per hour as a consequence of the $10.6 \mu \mathrm{m}$ wavelength [16], yielding a recoil heating rate of only $16 \mathrm{pK} / \mathrm{sec}$. At the background pressure of $<10^{-11}$ Torr, heating arising from diffractive background gas collisions [17,18 is $<5$ $\mathrm{nK} / \mathrm{sec}$. For the trap radial oscillation frequency of 6.6 $\mathrm{kHz}$, the intensity noise heating time constant is estimated to be $>2.3 \times 10^{4}$ seconds based on the measured laser intensity noise power spectrum [11]. A residual heating rate $<5 \mathrm{nK} / \mathrm{sec}$ is measured at full trap depth over 200 seconds. Trap 1/e lifetimes of 400 seconds are observed.

The $\mathrm{CO}_{2}$ laser trap is continuously loaded from a ${ }^{6} \mathrm{Li}$ MOT. The MOT is loaded from a Zeeman slower for 5 seconds, after which the MOT laser beams are tuned $\simeq 6 \mathrm{MHz}$ below resonance and lowered in intensity to $0.1 I_{\text {sat }}=0.25 \mathrm{~mW} / \mathrm{cm}^{2}$ to obtain a Doppler-limited temperature of $\simeq 150 \mu \mathrm{K}$ at a density of $10^{11} / \mathrm{cm}^{3}$. Following this loading stage, the MOT gradient magnets are extinguished and the upper $F=3 / 2$ hyperfine state is emptied to produce a 50-50 mixture of atoms in the lower $|F=1 / 2, M= \pm 1 / 2\rangle$ states $[7]$.

The $|F=1 / 2, M= \pm 1 / 2\rangle$ mixture is of particular interest, as it is predicted to exhibit a Feshbach resonance near $850 \mathrm{G}$ 19. A convenient feature of this mixture is that the s-wave scattering length vanishes in the absence of a bias magnetic field [19]. However, the scattering length varies between 0 and $-300 a_{0}$ as the bias magnetic field is tuned between 0 and $300 \mathrm{G}$ [19]. Hence, rapid evaporation can be turned on and off simply by applying or not applying a bias magnetic field.

The number of trapped atoms is enhanced by increasing the intensity of the $\mathrm{CO}_{2}$ laser during the loading stage 20. To accomplish this, the beam which emerges from the trap is recollimated after a ZnSe exit window by a $19.5 \mathrm{~cm}$ focal length ZnSe lens, and then retroreflected and orthogonally polarized using a rooftop mirror oriented at $45^{\circ}$ to the incoming polarization. The resulting backward-propagating beam is refocused into the trap region through the exit lens. After passing through the trap region, this beam is diverted by a thin film polarizer to a beam dump to avoid feedback into the laser. Typically $1.5 \times 10^{6}$ atoms are confined in the forward propagating trap beam alone. The backward-propagating beam increases this number to $3.5 \times 10^{6}$.

After the $\mathrm{CO}_{2}$ laser trap is loaded, the atoms are precooled by free evaporation. To initiate evaporative cooling, we apply a bias magnetic field of $130 \mathrm{G}$ by reversing the current in one of the MOT gradient coils, yielding a scattering length of $\simeq-100 a_{0}$. During free evaporation, a pneumatically controlled mirror slowly blocks the backward-propagating beam by diverting the power into a $100 \mathrm{~W}$ power meter. Since this beam is refocused, the trap region is Fourier-transform-related to the plane of the blocking mirror, and the trap smoothly evolves into a single beam configuration. After 6 seconds of free evaporation, the single beam trap contains $N=1.3 \times 10^{6}$ atoms at a temperature $T=50 \mu \mathrm{K}$. This precooling procedure provides excellent initial conditions for the forced evaporation experiments, since the resulting phase-space density for each state at full trap depth, $\rho_{i}=(N / 2)(h \nu)^{3} /\left(k_{B} T\right)^{3}$, is $8 \times 10^{-3}$, which is extremely high.

In all of our experiments, we characterize the velocity distribution of the trapped gas by time-of-flight imaging. We use the $\mathrm{A} / \mathrm{O}$ modulator to turn off the $\mathrm{CO}_{2}$ laser trap abruptly $(\Delta t<1 \mu \mathrm{s})$, permitting the gas to expand for a time between $400 \mu \mathrm{s}$ and $1.2 \mathrm{~ms}$ in zero bias magnetic field. Residual A/O leakage is reduced to less than $2 \times 10^{-5}$ of the maximum intensity by extinguishing the radio-frequency synthesizer output prior to the amplifier. Then a linearly polarized probe laser pulse with a resonant intensity of $0.1 I_{\text {sat }}$ and a detuning of 3 half linewidths $(\simeq 9 \mathrm{MHz})$ illuminates the gas for $10 \mu \mathrm{s}$. Simultaneously, a noncopropagating repumper, resonant with the D2 lines starting from the $F=3 / 2$ state, suppresses optical pumping into the upper $F=3 / 2$ hyperfine state. The probe detuning reduces sensitivity to the unresolved excited state hyperfine structure and light shifts from the resonant repumper. For the selected $9 \mathrm{MHz}$ detuning, the expansion time is chosen so that the imaged cloud has a small optical absorption $<35 \%$. An achromat at the vacuum system exit window produces a 1:1 image of the atomic distribution in an intermediate plane. This plane is imaged onto a CCD camera (Andor) using a microscope objective to produce a net magnification of $\simeq 4$. The magnification is calibrated by moving the axial position of the trap focus through \pm 1.25 $\mathrm{mm}$ using a micrometer-controlled translation stage. Fitting the central peak of the distribution to a straight line yields a magnification of 3.9.

The images are processed to obtain the transverse spatial distribution by integrating the measured optical depth in the axial direction. In typical measurements, the cloud expands ballistically by $100-200 \mu \mathrm{m}$ in $400 \mu \mathrm{s}$, much larger than its initial transverse dimension. In the classical regime, we assume ballistic expansion with a Maxwellian distribution. In this case, the temperature is readily determined from the transverse $1 / \mathrm{e}$ width of the cloud: $a(t)=v \sqrt{1 /\left(2 \pi \nu_{r}\right)^{2}+t^{2}}$, where $v=\sqrt{2 k_{B} T / M}$ is the thermal velocity and $t$ is the time. Since $\nu_{r}=6.6$ $\mathrm{kHz}$, for $t>>24 \mu \mathrm{s}, a(t)=v t$. Measurements of the cloud radius for several expansion times between $100 \mu \mathrm{s}$ and $600 \mu$ s fit very well to a straight line.

The number of atoms is determined from the spatially integrated optical depth of the absorption image and the absorption cross section $\sigma$. For each of the $M= \pm 1 / 2$ magnetic sublevels of the populated $F=1 / 2$ state, $\sigma$ 
is taken to be $\left(\lambda^{2} / \pi\right) /\left(1+(2 \Delta / \gamma)^{2}\right), 2 / 3$ of that of the cycling transition. Since the excited hyperfine states are unresolved compared to the linewidth $\gamma=5.9 \mathrm{MHz}$, this cross section contains contributions from both allowed transitions. Results for the number are consistent within $10 \%$ for several detunings $\Delta$ between 9 to $30 \mathrm{MHz}$ and -30 to $-9 \mathrm{MHz}$, and for variation of the camera focal plane over $\pm 1 \mathrm{~mm}$ from the plane which gives the sharpest image.

After precooling by free evaporation, further cooling is accomplished by lowering the trap depth, producing forced evaporation. We have developed scaling laws for the number of atoms, collision rate, and phase-space density as a function of trap depth $U$ for an optical trap which is continuously lowered [12]. These scaling laws are valid for a fixed $\eta=U /\left(k_{B} T\right)>>1$. For $\eta=10$, we find that the ratio of the final to initial phase-space density increases according to $\rho / \rho_{i}=\left(U_{i} / U\right)^{1.3}$. This result shows that lowering the trap depth by a factor of 100 should increase the phase-space density by a factor of 400 , producing a degenerate sample for $\rho_{i}>2.5 \times 10^{-3}$. To maintain a constant value of $\eta$, the trap should be lowered from its initial depth $U_{i}$ according to the formula

$$
U(t)=U_{i} /(1+t / \tau)^{\beta},
$$

which assures that the lowering rate slows as the collision rate decreases 12]. Taking $\eta=10$, we have $\beta=1.45$ and $1 / \tau=2.0 \times 10^{-3} \gamma_{i}$, where $\gamma_{i}$ is the initial elastic collision rate. For a 50-50 mixture of fermions, $\gamma_{i}=\pi N_{i} M \sigma \nu_{i}^{3} /\left(k_{B} T_{i}\right)$ with $N_{i}$ the initial total number of atoms. Note that $\gamma_{i}$ is reduced by a net factor of 4 compared to a single-component Bose gas with the same parameters. For a scattering length of $a \simeq-100 a_{0}$, the elastic cross section is $\sigma=8 \pi a^{2}=0.7 \times 10^{-11} \mathrm{~cm}^{2}$. Using $\nu_{i}=\left(\nu_{x} \nu_{y} \nu_{z}\right)^{1 / 3}=2.4 \mathrm{kHz}, N_{i}=1.0 \times 10^{6}$, and $T_{i}=50 \mu \mathrm{K}$, we obtain $\gamma_{i}=4.4 \times 10^{2} \mathrm{~s}^{-1}$ and $\tau=1.1 \mathrm{sec}$.

Unfortunately, the A/O modulator that controls the $\mathrm{CO}_{2}$ laser intensity produces an ellipticity which varies as the radio-frequency (rf) power is varied. The ellipticity is corrected at maximum rf power by a cylindrical telescope. However, the telescope provides only fixed compensation. Hence, as the rf power is decreased to lower the trap depth, the beam becomes elliptical, reducing $\nu_{x} \nu_{y} \nu_{z}$ by a factor of two compared to that expected on the basis of the laser power alone. Further, we find that the direction of the beam changes by $3 \mathrm{mrad}$ as the $\mathrm{rf}$ power is reduced by a factor of 100, causing vignetting. We align the trap beam to minimize this vignetting, but beam distortion still occurs. For this reason, we cannot accurately compare our evaporation results to the scaling law model. To compensate for the loss of confinement arising from the beam distortion as the trap is lowered, we increase $\tau$ to 3 seconds. The trap laser intensity is lowered using an Agilent (33120A) arbitrary waveform generator, the output of which is filtered with a time constant of $0.2 \mathrm{sec}$ before being applied to the multiplier input of the $\mathrm{A} / \mathrm{O}$ radio-frequency generator.

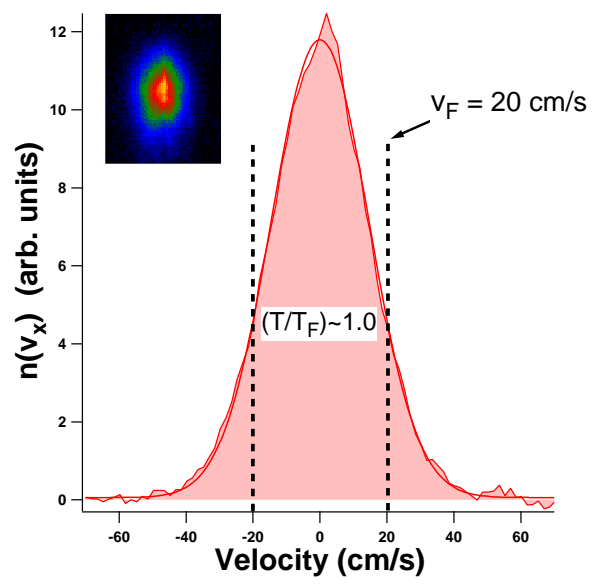

FIG. 1. Absorption image (inset) and velocity distribution after 10 seconds of forced evaporative cooling followed by recompression to full trap depth. An average of 5 trials is shown. $T / T_{F}=1$, as determined by a fit to a Maxwellian distribution. $v_{F}=20 \mathrm{~cm} / \mathrm{s}$ is the Fermi velocity for a total $N=8 \times 10^{5}$ atoms.

We have measured atomic velocity distributions after forced evaporative cooling for a variable time $t_{f}$. To provide a calibrated reference trap, time-of-flight images are recorded after adiabatic recompression to full trap depth over 11 seconds. This also increases the spatial density and hence the Fermi temperature, while preserving the phase-space density. Fig. 1 shows the velocity distribution for $t_{f}=10$ seconds. The total number of atoms remaining is $N=8 \times 10^{5}$, corresponding to a Fermi temperature of $T_{F}=h \nu(6 N / 2)^{1 / 3} / k_{B}=15 \mu \mathrm{K}$ for each state. Assuming a Maxwellian distribution, the gas is at a temperature of $15 \mu \mathrm{K}$, yielding $T / T_{F}=1$. At this temperature, a substantial number of atoms have velocities greater than the Fermi velocity of $20 \mathrm{~cm} / \mathrm{sec}$.

Near degeneracy, the energy of the atoms contains a contribution from the Fermi energy so that the true temperature is lower than that obtained using a MaxwellBoltzmann (MB) distribution which assumes that all of the energy is thermal. Hence, the low temperature absorption images are fit using a Thomas-Fermi (TF) approximation to determine $T / T_{F}$ [21,22], where the Fermi temperature $T_{F}$ is calculated using the measured trap frequencies and integrated atom number. At the lowest temperatures achieved in the experiments, the MB temperature is $\simeq 10 \%$ higher than the $\mathrm{TF}$ approximation.

Degeneracy is attained for $t_{f}=40$ seconds, where $T \simeq 5.8 \mu \mathrm{K}$ and $T / T_{F}=0.55$ with $3 \times 10^{5}$ atoms remaining. At this temperature, the gas is degenerate, and $\rho \simeq\left(T_{F} / T\right)^{3} / 6 \simeq 1$ [21]. We have also measured the temperature of the atoms in the lowered trap without recompression to full trap depth. We obtain temperatures a factor of $\simeq 10$ lower, i.e., $\simeq 580 \mathrm{nK}$, as expected for a harmonic trap which is lower in depth by a factor of $\simeq 100$.

Fig. 2 shows the velocity distribution for $t_{f}=60 \mathrm{sec}-$ onds. The total number of atoms is reduced to $10^{5}$, corre- 
sponding to a Fermi temperature of $8 \mu \mathrm{K}$. The measured temperature is below $4 \mu \mathrm{K}$, yielding $T / T_{F}=0.48$. Nearly all atoms have velocities less then the Fermi velocity of $14 \mathrm{~cm} / \mathrm{sec}$.

In the experiments, we achieve high evaporation efficiency $\chi \equiv \ln \left(\rho_{f} / \rho_{i}\right) / \ln \left(N_{i} / N_{f}\right)$ [23]. For example, after precooling, but prior to forced evaporation, $N_{i}=$ $1.3 \times 10^{6}$ and $\rho_{i}=8 \times 10^{-3}$ per state. After $40 \mathrm{sec}-$ onds of forced evaporation, $N_{f}=0.3 \times 10^{6}$ and $\rho_{f} \simeq 1$. Hence, $\chi \simeq 3.3$. The overall evaporation efficiency is similar. Starting with the loading conditions where the total number of atoms is $N_{i}=3.5 \times 10^{6}$ at a temperature of $150 \mu \mathrm{K}$, we obtain $\chi=2.9$ after 40 seconds of forced evaporation. Despite the trap distortion described above, these results are comparable to the best achieved in magnetic traps [23.

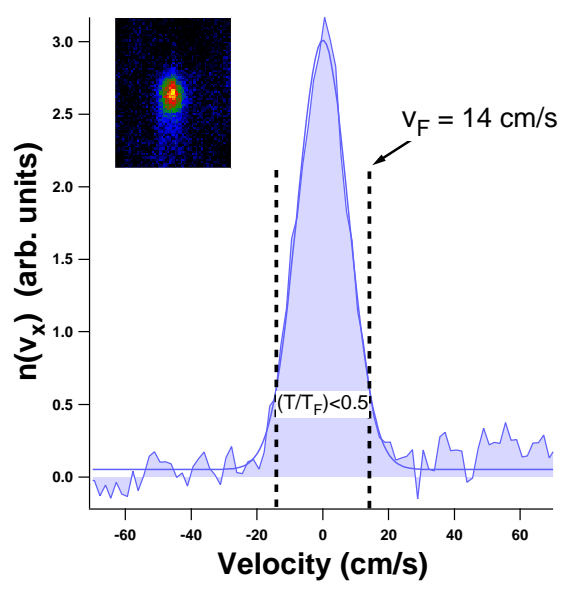

FIG. 2. Absorption image (inset) and velocity distribution after 60 seconds of forced evaporative cooling followed by recompression to full trap depth. An average of 5 trials is shown. $T / T_{F}<0.5$ as determined by a fit using a Thomas-Fermi approximation. $v_{F}=14 \mathrm{~cm} / \mathrm{s}$ is the Fermi velocity for a total $N=10^{5}$ atoms.

In conclusion, we have produced a degenerate, twocomponent ${ }^{6} \mathrm{Li}$ Fermi gas in a single beam all-optical trap by direct evaporative cooling. By using a stable $\mathrm{CO}_{2}$ laser trap at a background pressure of $<10^{-11}$ Torr, efficient evaporation over time scales of 85 seconds is achieved. In future experiments, it will be possible to attain scattering lengths of $\simeq-300 a_{0}$ by increasing the bias magnetic field to $300 \mathrm{G}$, thereby increasing the elastic cross section at low temperature by nearly factor of 10. This should enable preparation of a degenerate sample in just a few seconds, producing substantially lower temperatures by reducing the detrimental effects of any residual heating. We are currently preparing for a systematic study of the Feshbach resonance at higher magnetic field, and hope to observe superfluid pairing in a two-state Fermi gas.

This research is supported by the Physics divisions of the Army Research Office and the National Science
Foundation, the Fundamental Physics in Microgravity Research program of the National Aeronautics and Space Administration, and the Chemical Sciences, Geosciences and Biosciences Division of the Office of Basic Energy Sciences, Office of Science, U. S. Department of Energy.

[1] H. T. C. Stoof, M. Houbiers, C. A. Sackett, and R. G. Hulet, Phys. Rev. Lett. 76, 10 (1996); See also, M. Houbiers, et al., Phys. Rev. A 56, 4864 (1998).

[2] J. Bohn, Phys. Rev. A 61, 053409 (2000).

[3] M. Holland, S. J. J. M. F. Kokkelmans, M. L. Chiofalo, and R. Walser, Phys. Rev. Lett. 87, 120406 (2001).

[4] E. Timmermans, K. Furuya, P. W. Milonni, A. K. Kerman, Phys. Lett. A 285, 228 (2001).

[5] R. Combescot, Phys. Rev Lett. 83, 3766 (1999).

[6] M. Randeria, in Bose-Einstein Condensation, edited by A. Griffin, D. W. Snoke, and S. Stringari (Cambridge University Press, Cambridge, 1995), pp. 355-392.

[7] K. M. O'Hara, M. E. Gehm, S. R. Granade, S. Bali, and J. E. Thomas, Phys. Rev. Lett. 85, 2092 (2000).

[8] B. DeMarco, and D. S. Jin, Science 285, 1703 (1999).

[9] A. G. Truscott, K. E. Strecker, W. I. McAlexander, G. B. Patridge, and R. G. Hulet, Science, 291, 2570-2572 (2001).

[10] F. Schreck, L. Khaykovich, K. L Corwin, G. Ferrari, T. Bourdel, J. Cubizolles, and C. Salomon, Phys. Rev. Lett. 87, 080403 (2001).

[11] K. M. O'Hara, S. R. Granade, M. E. Gehm, T. A. Savard, S Bali, C. Freed, and J. E. Thomas, Phys. Rev. Lett. 82, 4204 (1999).

[12] K. M. O'Hara, M. E. Gehm, S. R. Granade, and J. E. Thomas, Phys. Rev. A 64, 051403(R) (2001).

[13] M. D. Barrett, J. A. Sauer, and M. S. Chapman, Phys. Rev. Lett. 87, 010404 (2001).

[14] S. Friebel, C. D'Andrea, J. Walz, M. Weitz, and T. W. Hänsch Phys. Rev. A 57, R20 (1998).

[15] We find by Monte-Carlo modeling that the parametric resonance frequency is shifted downward in a gaussian trap compared to that of a harmonic trap. The shift is temperature dependent and arises because the restoring force in a gaussian trap decreases as the radius increases. For an analytic treatment, see R. Jáuregui, Phys. Rev. A 64, 053403 (2001).

[16] T. Takekoshi and R. J. Knize, Opt. Lett. 21, 77 (1996).

[17] S. Bali, K. M. O'Hara, M. E. Gehm, S. R. Granade, and J. E. Thomas, Phys. Rev. A 60, R29 (1999).

[18] For a complete treatment of background gas collisioninduced heating including multiple scattering, see H. C. W. Beijerinck, Phys. Rev. A 62, 063614 (2000).

[19] M. Houbiers, H. T. C. Stoof, W. I. McAlexander, and R. G. Hulet, Phys. Rev. A 57, R1497 (1998).

[20] K. M. O'Hara, S. R. Granade, M. E. Gehm, and J. E. Thomas, Phys. Rev. A 63, 043403 (2001).

[21] D. A. Butts and D. S. Rokhsar, Phys. Rev. A 55, 4346 (1997). 
[22] B. DeMarco, Ph. D. Thesis (University of Colorado, Boulder, 2001).

[23] W. Ketterle and N. J. Van Druten, Adv. At. Mol. Opt. Phys. 37, 181 (1996). 